\title{
Learning to Predict Context-adaptive Convolution for Semantic Segmentation
}

\author{
Jianbo Liu ${ }^{1}$, Junjun $\mathrm{He}^{2}$, Jimmy S. Ren ${ }^{3}$, Yu Qiao ${ }^{2}$, and Hongsheng $\mathrm{Li}^{1}$ \\ 1 CUHK-SenseTime Joint Laboratory, The Chinese University of Hong Kong \\ 2 Shenzhen Key Lab of Computer Vision and Pattern Recognition, Shenzhen \\ Institutes of Advanced Technology, Chinese Academy of Sciences \\ 3 SenseTime Research
}

\{liujianbo@link, hsli@ee. cuhk.edu.hk

\begin{abstract}
Long-range contextual information is essential for achieving high-performance semantic segmentation. Previous feature re-weighting methods [34] demonstrate that using global context for re-weighting feature channels can effectively improve the accuracy of semantic segmentation. However, the globally-sharing feature re-weighting vector might not be optimal for regions of different classes in the input image. In this paper, we propose a Context-adaptive Convolution Network (CaC-Net) to predict a spatially-varying feature weighting vector for each spatial location of the semantic feature maps. In CaC-Net, a set of context-adaptive convolution kernels are predicted from the global contextual information in a parameter-efficient manner. When used for convolution with the semantic feature maps, the predicted convolutional kernels can generate the spatially-varying feature weighting factors capturing both global and local contextual information. Comprehensive experimental results show that our CaC-Net achieves superior segmentation performance on three public datasets, PASCAL Context, PASCAL VOC 2012 and ADE20K.
\end{abstract}

\section{Introduction}

Semantic segmentation aims at estimating a category label for each pixel of an input image, which is a fundamental problem in computer vision. It plays an important role in many applications including autonomous driving, image editing, computer-aided diagnosis, etc. Recently, state-of-the-art approaches leverage the Fully Convolutional Network (FCN) [23] as a base network to encode dense semantic representations from the input image and predict the class label for each pixel $[4,5,37,34,11,30]$. However, the significant scale variations of objects belonging to the same class and the similar appearances of objects belonging to different classes pose great challenges for semantic segmentation methods.

To overcome these challenges, many works have been proposed to exploit the long-range contextual information from the dense semantic representations $[4,37,34,11]$ to better distinguish scale and appearance ambiguity. One common solution is to aggregate the local context from different locations or subregions. The contextual information is collected by taking a fixed or an adaptively weighted aggregation from the local neighborhoods $[37,34,7,35,16]$. 
In contrast to encoding the relationship of local spatial context, another category of methods attempted to capture the global contextual information for estimating the channel-wise feature importances and using it to re-weight the feature channels for improving the segmentation accuracy. As illustrated in Figure 1 , the feature weighting can automatically highlight the features that are more relevant to the given scene and suppresses the irrelevant feature. For instance, boats usually appear in the sea or rivers but not in indoor environments. With the global context of a water scene, water-related feature channels should be higher weighted to increase the probability of predicting boat pixels. SE-Net [13] proposed the squeeze-excitation operation to learn a global feature weighting vector for weighting feature maps to improve image classification, detection and segmentation. EncNet [34] designed a global context encoding layer to weight features for improving semantic segmentation. Although this strategy has shown improved accuracy on segmentation, one of its key problems is that all the spatial locations share a common channel-wise weighting vector to calibrate the contributions of feature channels. For instance, features in the sky regions and the water regions of a water scene should be weighted differently.

To tackle this challenge, as shown in Figure 1, we propose a novel segmentation framework, which properly weights feature channels with the global context but in a spatially varying manner, i.e., feature channels at different spatial locations are modulated differently based on predictable and input-variant convolutions. Although there is a naive solution that simply generates kernels with a large number of parameters to conduct dot-product with the original feature maps, such strategies introduce too many parameters and cannot be used in practice. We propose a novel approach, which learns to predict Context-adaptive Convolution $(\mathrm{CaC})$ kernels from the global context for generating the spatiallyvarying feature weighting factors. To reduce the parameters and the computational burden, we do not predict all CAC kernel parameters with fully-connected (FC) layers as previous dynamic kernels [15] do, which need too many learnable FC parameters. We instead propose an efficient context-adaptive kernel learning scheme that generates the $\mathrm{CaC}$ kernel parameters via simple matrix multiplication. The $\mathrm{CaC}$ kernels not only fully encode global context of the input feature maps but also generate context-aware spatially-varying feature weighting factors for each spatial location via depth-wise convolution with the input feature maps. In addition, we utilize a series of dilated depth-wise convolutions with different dilation factors to effectively capture information of multiple scales.

Our main contributions can be summarized as threefold: (1) To better regularize the semantic segmentation with global contextual information, we propose to estimate spatially-varying feature weighting factors for properly weighting features at different spatial locations to improve the semantic segmentation performance. (2) We train an efficient deep network to predict the context-adaptive convolution kernels from the global context of the input image. The proposed $\mathrm{CaC}$ kernel prediction is both computational and memory efficient, which can effectively generate spatially-varying weights based on both global context and local information. (3) With the proposed $\mathrm{CaC}$ module, our proposed approach 
Previous methods for Feature Re-weighting

$\otimes:$ Element-wise multiplication

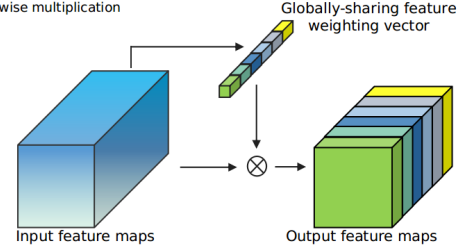

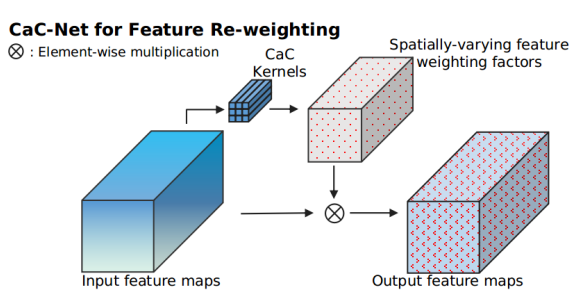

Fig. 1: (Left): Previous feature re-weighting methods [34,13] used a globally-sharing weighting vector to improve the performance of semantic segmentation, but ignores the spatially varying characteristics of the input image. (Right): Our CaC-Net learns to predict context-adaptive convolution kernels from the global context for weighting feature channels in a spatially-varying manner

achieves state-of-the-art performance on multiple public benchmarks, including PASCAL-Context, PASCAL VOC 2012, and ADE20K.

\section{Related Work}

Context aggregation. Fully convolution network [23] based methods have made great achievements in semantic segmentation. With a series of convolution and down-sampling operations, the features of deeper layers gradually capture information with larger receptive fields. However, they still have limited receptive fields and cannot effectively take advantages of the global or long-range context. Global or long-range contextual information aggregation has been shown their effectiveness on improving the segmentation accuracy of large homogeneous semantic regions or objects with large scale variations. ParseNet [20] proposed to capture the global context by concatenating a global pooling feature with the original feature maps. PSPNet [37] designed a Spatial Pyramid Pooling (SPP) module to collect contextual information of different scales. Atrous Spatial Pyramid Pooling (ASPP) [4,5] applied a set of different dilated convolutions to capture multi-scale contextual information. However, these methods treat all pixels in each sub-region with uniform or fixed weights for feature aggregation.

To achieve adaptive and flexible feature aggregations, APCNet [11] proposed an adaptive context module (ACM) to leverage local and global representations to estimate inter-pixel affinity weights for feature aggregation. CFNet [35] designed an aggregated co-occurrent feature (ACF) module to aggregate the co-occurrent context using the pair-wise similarities in the feature space. PSANet[38] aggregated contextual information for each pixel with a predicted attention map. DANet [7] proposed to apply a position attention module and channel attention module with the self-attention mechanism to aggregate features from spatial and channel dimensions respectively. These techniques show robustness to shape or scale variations of objects and are able to boost the segmentation performance. However, it is still challenging for this kind of methods to efficiently and accurately find all the pixels belonging to the same object class. 
Channel-wise feature re-weighting. To take advantages of the global contextual information of the input images, some pioneering methods $[13,34]$ have been proposed to re-weight different channels of the $2 \mathrm{D}$ feature maps with a scaling vector learned from the global context feature vector. Both SE-Net [13] and EncNet [34] learned a globally-sharing attention vector from the global context. SE-Net [13] proposed to learn feature weighting factors by the squeeze-excitation operation. The squeeze operation aggregates the feature maps across all spatial locations to produce a global context-encoded feature vector. Then the excitation operation learns the weighting factors from the global-context features. EncNet [34] predicted one globally-sharing feature re-weighting vector using a context encoding module. This module integrates dictionary learning and residual encoding components to learn a global context encoded feature vector, based on which, the feature weighting factor vector is predicted. However, both methods only consider the global context and output a feature re-weighting vector that is shared across all spatial locations. Such a globally-sharing weighting scheme might not be suitable for different spatial regions belonging to different objects in the same scene. To tackle this challenge, we propose to predict learnable kernels for re-weighting the feature maps in a spatially-varying manner.

Dynamic filters. Dynamic filters or kernels were proposed by [15] to generate content-aware filters, which are conditional on the input images. These filters are adaptive to the input and are predicted by the neural network. It has shown effectiveness in various computer vision applications. Some methods adopted the predicted dynamic filters for low-level vision and video understanding, e.g., video interpolation [26] and image denoising [24]. Pixel-Adaptive Convolution (PAC) Network [29] was proposed to predict spatially-varying kernels for several computer vision applications including deep joint image upsampling, semantic segmentation and efficient CRF inference. SAC [36] utilized a scale regression layer to predict position-adaptive scale coefficients, which were used to automatically adjust the sizes of receptive fields for objects of different sizes. DMNet [10] exploited a set of dynamic filters of different sizes, which were generated from multi-scale neighborhoods for handling the scale variations of objects for semantic segmentation. The previous methods with dynamic filters directly predict all the filter parameters, which are time inefficient and occupy too much memory. In comparison, our work learns to predict the context-adaptive convolution kernels from global context in a parameter-efficient manner.

\section{Method}

Feature re-weighting has proven to be a powerful approach to capture long-range semantic context by learning channel-wise weighting factors from the global contextual information. Although it has shown its effectiveness in various tasks including classification, detection and semantic segmentation [13,34], one of its key issues is that the weighting vector is shared by all spatial locations of the $2 \mathrm{D}$ feature map. 


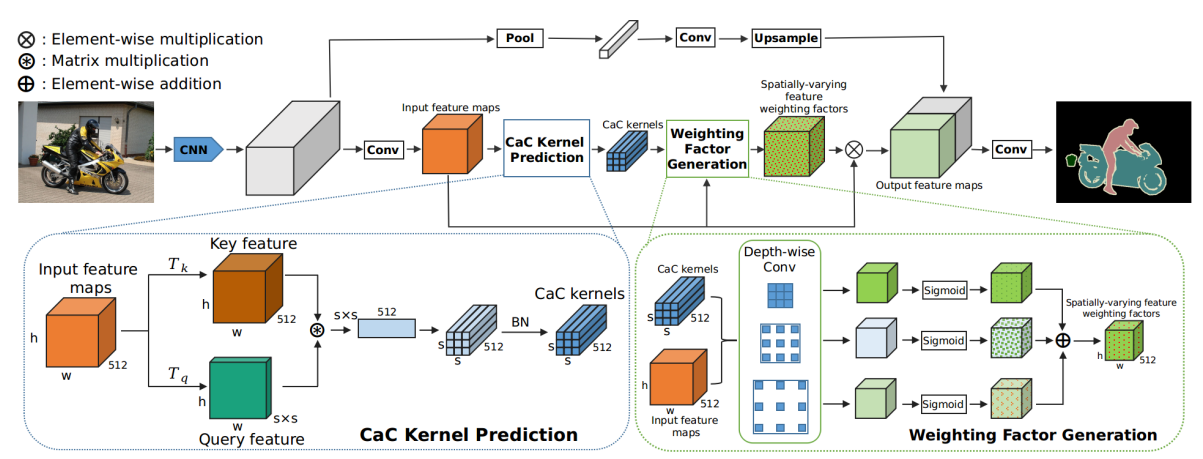

Fig. 2: Pipeline of Context-adaptive Convolution Network (CaC-Net). CaC-Net consists of a backbone convolution neural network (CNN), a Context-adaptive Convolution $(\mathrm{CaC})$ kernel prediction module and a spatially-varying weight generation module. The key component, CaC kernel prediction, learns to predict a spatially-sharing contextaware convolution kernels. The weight generation module uses the predicted dynamic kernels to generate one spatially-varying feature weighting factor for each location in the input feature maps

We argue that, for semantic segmentation, a globally-shared weighting vector is not an optimal solution, as different spatial locations generally belong to objects of different classes. Therefore, desirable feature weighting factors should still be learned from the global context but be spatially-varying to capture different locations' unique characteristics to achieve high segmentation accuracy. Predicting convolution kernels from the global context to generate the spatially-varying weights is a desirable solution. Compared with traditional convolutions on the feature maps, which only have local receptive fields and are input-invariant, predicted kernels from the global context are well aware of the overall scene structure for better weighting the features at different locations.

To achieve this goal, we propose a novel Context-adaptive Convolution $(\mathrm{CaC})$ Network (see Fig. 2), which consists of a 2D Convolutional Neural Network (CNN)-based backbone for encoding the input images into $2 \mathrm{D}$ feature maps and a Context-adaptive Convolution $(\mathrm{CaC})$ module for learning to channel-wisely re-weight the $2 \mathrm{D}$ feature maps with global context. A series of context-adaptive convolution kernels are predicted from the $\mathrm{CaC}$ module. Unlike the previous dynamic filters, which require using a large number of learnable parameters to predict the kernels, the proposed $\mathrm{CaC}$ kernels are predicted in a parameter-efficient manner via simple matrix multiplication. Importantly, the CaC kernels are predicted from global context of the $2 \mathrm{D}$ feature maps. The convolution with the $\mathrm{CaC}$ kernels can well integrate the global context as well as the multi-scale information of the $2 \mathrm{D}$ feature maps to predict the spatially-varying feature re-weighting factors. The proposed framework achieves state-of-the-art segmentation accuracy on multiple public benchmarks with small computational overhead. 


\subsection{Context-adaptive Convolution Kernel Prediction}

We adopt a pre-trained ResNet [12] with the dilated convolutions as the backbone of our segmentation framework. Following [33,4,34], we remove the downsampling operations and set the dilation rates to be 2 and 4 at the last two ResNet-blocks to generate an output feature map of $1 / 8$ spatial size of the input image. It encodes each input 2D image into a 2D feature map $X \in \mathbb{R}^{h \times w \times c}$, where $h, w, c$ are the height, width, and feature channels of the feature map. The feature map can roughly capture semantic information of the input image. Properly re-weighting the feature map $X$ in a sptially-varying manner according to the global context can boost the segmentation performance.

To conduct spatially-varying feature re-weighting via kernel prediction, there are two naive solutions: (1) one can directly predict $c$ sets of $s \times s \times c$ convolutional kernels (where $s \times s$ is the kernel spatial size) following dynamic filters [15] and then conducting convolution of the input feature map $X$ with the kernels to generate the $c$ sets of feature weighting maps. However, this solution requires a fully connected (FC) layer to predict the kernel weights, which has too many $\left(s^{2} c^{3}\right)$ learnable parameters and computation cost. (2) One can also predict the kernels from the global average pooled features of the input feature map with an FC layer to capture the global context. Feature weighting maps can be obtained by conducting convolution on the input feature map with the predicted kernels. However, such a solution loses all spatial information during kernel prediction and show inferior performance in our experiments.

To tackle the challenges, our proposed Context-adaptive Convolution module predicts a series of $s \times s \times c \mathrm{CaC}$ convolution kernels from the global context in a parameter-efficient manner. The input feature map $X \in \mathbb{R}^{h \times w \times c}$ is first transformed into the $2 \mathrm{D}$ query feature map $Q \in \mathbb{R}^{h \times w \times s^{2}}$ and the key feature map $K \in \mathbb{R}^{h \times w \times c}$ by two independent transformations $T_{k}$ and $T_{q}$, respectively. The transformations $T_{k}$ and $T_{q}$ are independently implemented by $1 \times 1$ convolutions. Generally, the key feature $K \in \mathbb{R}^{h \times w \times c}$ captures $c$ different characteristics of the input feature map $X$ via its $c$-dimensional feature maps, while the query feature $Q \in \mathbb{R}^{h \times w \times s^{2}}$ is used to capture the global spatial distributions of $K$, where $s^{2}$ sets of global spatial characteristics would be captured by $Q$.

To achieve the goal, the query feature and key feature are first reshaped to obtain $\bar{Q} \in \mathbb{R}^{n \times s^{2}}$ and $\bar{K} \in \mathbb{R}^{n \times c}$, where $n=h \times w$. For the $i$ th column $\bar{Q}(:, i) \in$ $\mathbb{R}^{n}$ of query feature, it can be used to capture the overall spatial distribution of each feature channel $j$ of the key feature $\bar{K}(:, j) \in \mathbb{R}^{n}$ over all $n=h \times w$ spatial locations via the dot product $\langle Q(:, i), K(:, j)\rangle$. The result would be a scalar to measure the similarity between the spatial distributions $Q(:, i)$ and $K(:, j)$. If we repeat the procedure for all $c$ feature channels of $K$, we obtain a $c$-dimensional

vector to characterize the spatial distributions of each feature channel of $\bar{K}$ with a query vector $\bar{Q}(:, i)$. Since we have $s^{2}$ query vectors in total, we can capture $s^{2}$ characteristics of the overall spatial distributions of the $c$-dimensional key feature map $K$ as

$$
\bar{D}=\bar{Q}^{\top} \bar{K},
$$

where $\bar{D} \in \mathbb{R}^{s^{2} \times c}$. 
We then reshape $\bar{D}$ into the size of $s \times s \times c$ and use a batch normalization to modulate it to obtain the predicted $D \in \mathbb{R}^{s \times s \times c}$ as our CaC kernels, which are used to convolved with the input feature map $X$ to generate the spatially-varying feature re-weighting factors for all $h \times w$ spatial locations.

Note that the $\mathrm{CaC}$ kernels are predicted and would therefore be adaptive to different inputs to capture their different global context. There are two distinct advantages of the predicted convolution kernel $D$. 1) The CaC kernels are able to capture the global context of the input feature map $X$, since the matrix multiplication in Eq. (1) considers all spatial locations for kernel prediction. 2) The $\mathrm{CaC}$ kernels are generated in a parameter-efficient manner, where only $c^{2}+s^{2} c$ learnable parameters are needed with an inference time complexity of $O\left(c^{2}+s^{2} c+s c\right)$ for kernel prediction, which is significantly lower than $O\left(s^{2} c^{3}\right)$ parameters and complexity of dynamic filters.

\subsection{Spatially-varying Weight Generation}

We use the predicted $\mathrm{CaC}$ kernels to produce the spatially-varying weighting map for weighting each pixel of the input feature maps. The predicted kernels $D \in \mathbb{R}^{s \times s \times c}$ are used in a depth-wise convolution. Therefore, each channel of $D$ is responsible for modulating one channel of the input feature maps independently. To further encourage the predicted kernel $D$ being scale invariant as well as capturing multi-scale context of the input feature map. We denote the original predicted kernels $D$ with dilation 1 as $D_{1}$, and create another two CaC kernels with shared parameters of $D$ but with different dilation rates 3 and 5 , which are denoted as $D_{2}$ and $D_{3}$.

As shown in Figure 2, for each set of predicted CaC kernels of $D_{1}, D_{2}, D_{3}$, they are used to separately perform depth-wise convolution on the input feature map $X$ followed by the sigmoid function. Each of them would generate an independent spatially-varying weighting map $W_{1}, W_{2}, W_{3} \in \mathbb{R}^{h \times w \times c}$, which are averaged to generate the overall spatially-varying weighting map $W$,

$$
W=\frac{1}{3}\left(W_{1} \oplus W_{2} \oplus W_{3}\right),
$$

where the symbol $\oplus$ denotes the element-wise addition.

Given $W$, we re-weight the input feature maps as $X^{\star}=X \odot W$, where $\odot$ represents the element-wise multiplication. In this manner, we develop a computationally efficient way to predict the spatially-varying feature weighting factors for each spatial location of the input feature map $X$. For each predicted feature weighting vector at the spatial location $W(i, j) \in \mathbb{R}^{c}$, it re-weights the features $X(i, j)$ according to the global contextual information. Intuitively, the larger values in the $W(i, j)$ vector highlight more target class-related features while the smaller values suppress non-target-class-related features according to the global context. 


\subsection{Global Pooling and Multi-head Ensembles}

Following state-of-the-art segmentation frameworks [35,37], we also integrate a global pooling branch in our framework, which globally averages the feature vectors of all spatial locations and replicates the vector to all spatial locations. This replicated global-pooling feature maps are channel-wise concatenated with the re-weighted feature maps $X^{\star}$ to obtain the final feature maps for segmentation.

The multi-head ensembling strategy is implemented by performing multiple proposed $\mathrm{CaC}$ modules in parallel and producing multiple output feature maps. Since the different head modules operate on the different subspace of the input feature maps, some previous works $[35,10,31]$ show that the multi-head ensembling strategy is able to further increase the performance. In our work, we apply this strategy to improve the capability of our CaC-Net by concatenating two output feature maps from our $\mathrm{CaC}$ modules.

\section{Experiments}

In this section, we first introduce the implementation details, training strategies and evaluation metrics of the experiments. Then, to evaluate the proposed CaCNet, we conduct comprehensive experiments on three public datasets, Pascal Context [25], Pascal VOC 2012 [6] and ADE20K [40]. The ablation study of our CaC-Net is carried out on the Pascal Context dataset. Finally, we report the overall results on PASCAL Context, PASCAL VOC 2012 and ADE20K.

\subsection{Implementation Details}

Network Structure. We adopt ResNet [12] as our backbone. The stride of the last two stages of the backbone networks is removed and these dilation rates are set as 2 and 4 respectively. Thus the size of the feature maps is $8 \times$ smaller than that of the input image. To predict a semantic label for each pixel, the output of our CaC-Net is upscaled to the size of input image by bilinear interpolation. The ImageNet [28] pre-trained weights are adopted to initialize the backbone networks.

Training Setting. A poly learning rate policy [4], $l r=$ initial_lr $\times(1-$ $\left.\frac{\text { iter }}{\text { total_iter }}\right)^{\text {power }}$ is used. We set the initial learning rate as 0.001 for PASCAL Context [25], 0.002 for PASCAL VOC 2012 [6] and 0.004 for ADE20K [40]. The power of poly learning rate policy is set as 0.9 . The optimizer is stochastic gradient descent (SGD) [3] with momentum 0.9 and weight decay 0.0001 . We train our CaC-Net for 120 epochs for PASCAL Context dataset, 80 epochs for PASCAL 2012 dataset and 180 epochs for ADE20K dataset. We set the crop size to $512 \times 512$ on PASCAL Context and PASCAL 2012. Due to the average image size is larger than the other two datasets, we use $576 \times 576$ as the crop size on ADE20K. For data augmentation, we only randomly flip the input image and scale it randomly in the range from 0.5 to 2.0. As the prior work [34,35], we adopt an auxiliary segmentation loss, which is added after Res-4. We adopt Sync-BN [34] for normalization and set the batch size as 16 for all experiments. 
Table 1: Ablation study of CaC-Net on PASCAL Context dataset. CaC represents our proposed $\mathrm{CaC}$ module. $\mathrm{GP}$ indicates the global pooling

\begin{tabular}{llll|l}
\hline Method Backbone CaC GP pixAcc\% & mIoU\% \\
\hline \hline FCN & ResNet50 & & 76.0 & 45.8 \\
CaC-Net ResNet50 & $\checkmark$ & & 79.8 & 52.0 \\
CaC-Net ResNet50 & $\checkmark$ & $\checkmark$ & 80.2 & 52.5 \\
CaC-Net ResNet101 & $\checkmark$ & $\checkmark$ & 81.5 & 55.4 \\
\hline
\end{tabular}

Table 2: Ablation study of different setting in training and evaluation strategies. DS: Deep supervision loss strategy [37]. Flip: horizontally flipping the input image for evaluation. MS: Multi-scale evaluation

\begin{tabular}{ccccc|c}
\hline \multicolumn{3}{l}{ Backbone DS Flip MS pixAcc\% } & mIoU\% \\
\hline \hline ResNet50 & & & & 79.4 & 50.7 \\
ResNet50 & $\checkmark$ & & & 79.7 & 51.5 \\
ResNet50 & $\checkmark$ & $\checkmark$ & & 79.9 & 51.8 \\
ResNet50 & $\checkmark$ & $\checkmark$ & $\checkmark$ & 80.2 & 52.5 \\
\hline
\end{tabular}

Evaluation Metrics. We choose the standard evaluation metrics of pixel accuracy (pixAcc) and mean Intersection of Union (mIoU) as the evaluation metrics in this experiments. Following the best practice $[34,11,7]$, we apply the strategy of averaging the network predictions in multiple scales for evaluation. For multi-scale evaluation, we first resize the input image to multiple scales and horizontally flip them. Then the predictions are averaged as final predictions.

\subsection{Results on PASCAL Context}

PASCAL Context dataset [25] is a challenging scene understanding dataset, which provides the semantic labels for the images. There are 4,998 images for training and 5,105 images for validation on PASCAL Context dataset. Following previous work $[34,35]$, the 59 most frequent categories are used for training our CaC-Net and all the other classes are considered as the background class.

Ablation study on CaC-Net. We conduct experiments with different settings to evaluate the performance of our proposed CaC-Net using a ResNet-50 [12] backbone on the PASCAL Context dataset. The baseline is a ResNet-50 based FCN [23] by removing the proposed $\mathrm{CaC}$ modules and the global pooling branch in our CaC-Net. We choose the pixAcc and mIoU for 60 classes on the PASCAL Context dataset as our evaluation metrics for the ablation study.

We first evaluate the individual components, which are added into the baseline FCN one at a time. The size of predicted $\mathrm{CaC}$ kernels is $3 \times 3$ and we use two $\mathrm{CaC}$ modules in all experiments. As shown in Table 1, the baseline FCN with a ResNet50 backbone achieves $76.0 \%$ pixAcc and $45.8 \%$ mIoU. With our proposed $\mathrm{CaC}$, the results of pixACC and mIoU are increased by $3.8 \%$ and $6.2 \%$ respectively. The global pooling branch results in a further $0.5 \% \mathrm{mIoU}$ improvement. We can see that our proposed $\mathrm{CaC}$ module can significantly improve the segmentation results.

We also conduct experiments to explore training and evaluation strategies, other plausible ways of generating the weighting maps, the influence of the number of our proposed $\mathrm{CaC}$ modules for feature concatenation as introduced in Sec. 3, the kernel size of our predicted $\mathrm{CaC}$ kernels, and the dilation rates in the feature weighting factor generation.

Ablation study on training and evaluation strategies. We conduct experiments to explore the effects of training and evaluation strategies and the results 
Table 3: The results of dif- Table 4: The results of dif- Table 5: The results of ferent numbers of our $\mathrm{CaC}$ ferent kernel sizes used in different dilation rate sets module exploited in the our proposed $\mathrm{CaC}$ module for generating the weighting CaC-Net

\begin{tabular}{|c|c|c|c|c|c|c|c|}
\hline & \multirow{2}{*}{\multicolumn{3}{|c|}{$H=1 H=2 H=3 H=4$}} & \\
\hline & & & & $\overline{A c c}$ & 80 & 80 & 80.1 \\
\hline $\begin{array}{l}\text { pixAcc } \\
\text { mIoU }\end{array}$ & $\begin{array}{ll}79.9 & 80.2 \\
51.7 & 52.5\end{array}$ & $\begin{array}{l}79.9 \\
52.3\end{array}$ & & $\mathrm{mIoU}$ & 52.5 & 52.3 & \\
\hline
\end{tabular}

factors in $\mathrm{CaC}-\mathrm{Net}$

\begin{tabular}{c|cccc}
\hline & $\{1\}$ & $\{1,2\}$ & $\{1,2,3\}$ & $\{1,2,3,4\}$ \\
\hline \hline pixAcc & 79.9 & 80.1 & 80.2 & 79.7 \\
mIoU & 51.9 & 52.1 & 52.5 & 51.6 \\
\hline
\end{tabular}

Table 6: Results of alternative ways to generate weighting map and dynamic kernels.

\begin{tabular}{c|ccc|cc|c}
\hline & Fixed & FC & GAP & \multicolumn{3}{|c|}{ SEHead } \\
\hline \hline pixAcc & 79.7 & 79.8 & 79.8 & 79.7 & 79.7 & 80.0 \\
mIoU & 51.3 & 51.5 & 51.6 & 50.9 & 51.2 & 52.5 \\
\hline
\end{tabular}

Table 7: Parameters and FLOPS of backbone and segmentation heads from APCNet [11], DMNet [10], and our CaC-Net.

\begin{tabular}{c|cccc}
\hline Methods & \multicolumn{3}{|c}{ Backbone APCNet DMNet } & Ours \\
\hline \hline Parameters & $56 \mathrm{M}$ & $+10 \mathrm{M}$ & $+9 \mathrm{M}$ & $+5 \mathrm{M}$ \\
FLOPS & $225 \mathrm{G}$ & $+22 \mathrm{G}$ & $+20 \mathrm{G}$ & $+21 \mathrm{G}$ \\
\hline
\end{tabular}

are shown in Table 2. We use a deep supervision strategy by adding an FCN head to the output of ResNet- 4 as an auxiliary loss. From the table, we observe that the deep supervision training strategy results in a $0.8 \%$ mIOU improvement. We adopt the image flipping and multi-scale evaluation strategy during inference. These two evaluation strategies can further boost the performance of segmentation.

\section{Ablation study on the alternative ways to generate weighting maps} and dynamic kernels. To investigate other possible ways to generate spatiallyvarying weighting maps, we keep our overall framework unchanged but replace our $\mathrm{CaC}$ modules with alternative approaches to generate the feature weighting maps. The first intuitive way is to directly predict the feature weighting maps via traditional convolutions on the input feature maps, whose parameters are still learned during training but are fixed to different inputs during testing. If our $\mathrm{CaC}$ kernels are replaced by the input-invariant convolutions of the same kernel sizes and different dilation rates. Denoted by "Fixed" in Table 6, the performance of the fixed-kernel channel weighting prediction declines dramatically, which demonstrates that the predicted $\mathrm{CaC}$ kernels can generate context-aware feature weighting maps to boost the performance of semantic segmentation.

Meanwhile, there have some other possible ways to predict the dynamic filters. If we directly generate the dynamic kernel weights by one FC layer, the parameter overhead of this FC layer is $h \times w \times s^{2} \times c^{2}$, which are about $h w$ times more parameters than our method and occupy too much GPU memory to be implemented in practice. If we instead adopt a depth-wise FC layer on the input feature map to generate the dynamic kernel weights. The parameters of the depth-wise FC layer reduce to $h \times w \times s^{2} \times c$, which are the same as our $\mathrm{CaC}$ module. However, the mIoU on PASCAL Context decreases to $51.7 \%$ ("FC" in Table 6) with a ResNet-50 backbone, which is lower than our CaC-Net (52.5\%).

The third alternative way is to use the global average pooling (GAP) features of the input feature maps to predict the dynamic kernels. In this way, the dynamic kernels can also capture global context. The input feature maps is first average pooled to a $c$-d vector followed by an FC layer to predict the $s \times s \times c$ dynamic kernels. However, the parameters of this scheme would be 
$s^{2} c^{2}$, significantly more than our CaC module's $c^{2}+s^{2} c$ parameters. In addition, since the input feature map is average pooled first, the image spatial structure is lost, which limits its capacity on predicting spatially-aware dynamic kernels. If replacing our $\mathrm{CaC}$ kernels with such dynamic kernels from GAP features, the mIoU on PASCAL Context decreases to 51.9\% ("GAP" in Table 6) on PASCAL Context.

\begin{abstract}
Ablation study on comparison with the globally-sharing feature reweighting methods. To compare with globally-sharing feature re-weighting methods, we conduct two experiments by replacing the proposed $\mathrm{CaC}$ modules in our CaC-Net with SE-Head [13] and EncHead [34] for globally-sharing feature re-weighting. The results are shown in Table 6 , which demonstrate the proposed spatially-varying feature re-weighting method shows superior performance than the globally-sharing feature re-weighting methods for semantic segmentation.
\end{abstract}

Ablation study on the number of $\mathbf{C a C}$ modules. Table 3 illustrates the effects of different number of $\mathrm{CaC}$ modules for multi-head ensemble in our proposed $\mathrm{CaC}-\mathrm{Net}$, which shows that two $\mathrm{CaC}$ modules is optimal for achieving the best performance for semantic segmentation for our designed CaC-Net. The results are getting worse when $H$ is larger than 2 because the larger $H$ introduces additional parameters and model capacity, which might overfit the data if $\mathrm{H}$ is too large. We therefore fix the number of $\mathrm{CaC}$ module to be 2 .

Ablation study on kernel size. In following experiments, the influence of the kernel size of the predicted dynamic $\mathrm{CaC}$ kernels is investigated. We report results of three sets of kernel sizes, $3+3,3+5,3+7$, of the two CaC modules respectively in Table 4 . We first set the kernel size of one $\mathrm{CaC}$ module as $3 \times 3$ according to the experience that $3 \times 3$ kernels is usually sufficient to obtain good performance and is computationally efficient. By increasing the kernel size of another $\mathrm{CaC}$ module from $3 \times 3,5 \times 5,7 \times 7$, we observe that the performance of our proposed $\mathrm{CaC}$-Net decreases slightly. The reason might be increasing the kernel size also requires many additional FC parameters, which causes the models to be difficult to train. To reduce the computational cost and achieve the optimal performance, we set the kernel size of both $\mathrm{CaC}$ modules to $3 \times 3$.

Ablation study on dilation rates. For the spatially-varying feature weighting factor generation, we exploit a set of dilated depth-wise convolutions in each predicted $\mathrm{CaC}$ kernel to increase the ability of capturing multi-scale contextual information. The different dilation rates are explored here. Table 5 shows the results of different dilation rates adopted in our CaC-Net. We found that the dilated convolution could boost the performance of our proposed CaC-Net as the dilated convolution increase the receptive fields. However, the performance of the dilated rate sets $\{1,2,3,4\}$ is lower than that of $\{1,2,3\}$ because of two aspects: (a) The receptive field of the dilation rate 3 is enough for the size of feature maps in our proposed CaC-Net for the PASCAL Context dataset. (b) Too many dilation rates bring too large computational complexity. Based on this observation, the dilation set that we choose is $\{1,2,3\}$ in all experiments.

Comparisons with the state-of-the-art methods. The results are shown in Table 8. APCNet [11] and CFNet [35] both exploit a dynamic or adaptive 
Table 8: Segmentation results of stateof-the-art methods on PASCAL Context dataset

\begin{tabular}{lll}
\hline Method & Backbone & mIoU\% \\
\hline \hline FCN-8S [23] & & 37.8 \\
CRF-RNN [39] & 39.3 \\
ParseNet [21] & 40.4 \\
HO_CRF [1] & 41.3 \\
Piecewise [19] & & 43.3 \\
DeepLab-v2 [4] & ResNet101-COCO 45.7 \\
RefineNet [18] & ResNet152 & 47.3 \\
MSCI [17] & ResNet152 & 50.3 \\
EncNet [34] & ResNet101 & 51.7 \\
DANet [7] & ResNet101 & 52.6 \\
APCNet [11] & ResNet101 & 54.7 \\
CFNet [35] & ResNet101 & 54.0 \\
ACNet [8] & ResNet101 & 54.1 \\
APNB [41] & ResNet101 & 52.8 \\
DMNet [10] & ResNet101 & 54.4 \\
\hline Ours & ResNet50 & 52.5 \\
Ours & ResNet101 & $\mathbf{5 5 . 4}$ \\
\hline
\end{tabular}

Table 9: Segmentation results of stateof-the-art methods on ADE20K validation set

\begin{tabular}{lll}
\hline Method & Backbone & mIoU\% \\
\hline \hline FCN [23] & & 29.39 \\
SegNet [2] & & 21.64 \\
RefineNet [18] & ResNet152 & 40.7 \\
PSPNet [37] & ResNet101 & 43.29 \\
EncNet [34] & ResNet101 & 44.65 \\
SAC [36] & ResNet101 & 44.30 \\
PSANet [38] & ResNet101 & 43.77 \\
UperNet [40] & ResNet101 & 42.66 \\
APCNet [11] & ResNet101 & 45.38 \\
CFNet [35] & ResNet101 & 44.89 \\
CCNet [14] & ResNet101 & 45.22 \\
APNB [41] & ResNet101 & 45.24 \\
ACNet [8] & ResNet101 & 45.90 \\
DMNet [10] & ResNet101 & 45.50 \\
\hline Ours & ResNet101 & $\mathbf{4 6 . 1 2}$ \\
\hline
\end{tabular}

manner to globally aggregate features, which has expensive computational and memory cost. Our proposed CaC-Net with ResNet101 backbone surpasses other approaches by significant margins with a light-weight head. Meanwhile, the performance of CaC-Net with ResNet50 backbone even achieves better performance than most previous methods with a deeper backbone. Especially, by learning to predict the spatially-varying feature weighting factors for each location, the performance of our proposed CaC-Net significantly outperforms EncNet [34], which predicts a globally-sharing weight factor for re-weighting feature maps. In Figure 3 , we illustrate the visual improvements of our approach over the baseline FCN network on the PASCAL Context. It can be seen that our proposed method boosts the performance of semantic segmentation in some challenging scenarios.

Meanwhile, with the dilated ResNet-101 backbone and input image size $512 \times$ 512 , the computational and parameter overheads of the backbone, the heads of APCNet, DMNet and our CaC-Net are provided in Table 7. Compared with the backbone, the proposed CaC-Net brings less than 10\% extra cost. Compared with the two other segmentation heads with two times more parameters, our CaC-Net has a $>1 \%$ mIoU improvement on the PASCAL Context dataset.

\subsection{Results on PASCAL VOC 2012}

The PASCAL VOC 2012 dataset [6] is one of the most competitive semantic segmentation benchmarks, which contains 20 foreground object classes and 1 background class. There are 1, 464 images for training, 1, 449 images for validation and 1, 456 images for testing in the original PASCAL VOC 2012 dataset. Following the best practice $[34,35,11]$, the augmented training set of PASCAL VOC2012 [9] is adopted as our training set, which includes 10,582 training images. The training strategy is the same as [34,35,11], we first train our CaC-Net on the augmented training set, and then fine-tune on the original training and 

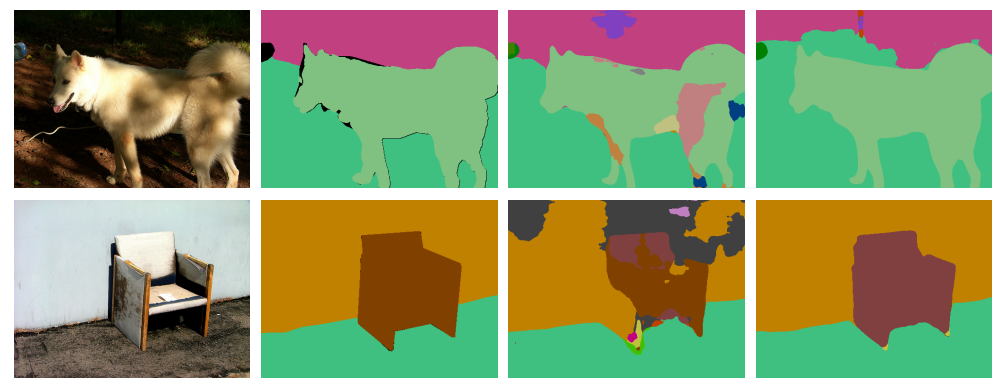

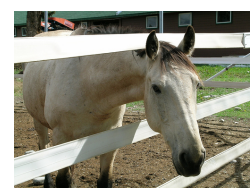

(a) Image

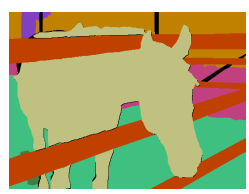

(b) GT

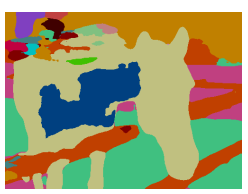

(c) Baseline

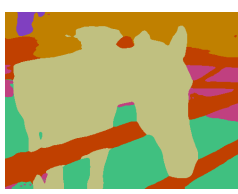

(d) CaC-Net

Fig. 3: Visualization examples from the PASCAL Context dataset

Table 10: Results of each category on PASCAL VOC 2012 test set. Our CaC-Net achieves $85.1 \%$ without MS COCO dataset pre-training.

\begin{tabular}{l|ccccccccccccccccccccc}
\hline Method & aero bike bird & boat bottle bus & car & cat & chair & cow & table & dog & horse mbike person plant sheep sofa train & tv & mIoU\% \\
\hline \hline FCN [23] & 76.8 & 34.2 & 68.9 & 49.4 & 60.3 & 75.3 & 74.7 & 77.6 & 21.4 & 62.5 & 46.8 & 71.8 & 63.9 & 76.5 & 73.9 & 45.2 & 72.4 & 37.4 & 70.9 & 55.1 & 62.2 \\
DeepLabv2 [4] & 84.4 & 54.5 & 81.5 & 63.6 & 65.9 & 85.1 & 79.1 & 83.4 & 30.7 & 74.1 & 59.8 & 79.0 & 76.1 & 83.2 & 80.8 & 59.7 & 82.2 & 50.4 & 73.1 & 63.7 & 71.6 \\
CRF-RNN [39] & 87.5 & 39.0 & 79.7 & 64.2 & 68.3 & 87.6 & 80.8 & 84.4 & 30.4 & 78.2 & 60.4 & 80.5 & 77.8 & 83.1 & 80.6 & 59.5 & 82.8 & 47.8 & 78.3 & 67.1 & 72.0 \\
DeconvNet [27] & 89.9 & 39.3 & 79.7 & 63.9 & 68.2 & 87.4 & 81.2 & 86.1 & 28.5 & 77.0 & 62.0 & 79.0 & 80.3 & 83.6 & 80.2 & 58.8 & 83.4 & 54.3 & 80.7 & 65.0 & 72.5 \\
DPN [22] & 87.7 & 59.4 & 78.4 & 64.9 & 70.3 & 89.3 & 83.5 & 86.1 & 31.7 & 79.9 & 62.6 & 81.9 & 80.0 & 83.5 & 82.3 & 60.5 & 83.2 & 53.4 & 77.9 & 65.0 & 74.1 \\
Piecewise [19] & 90.6 & 37.6 & 80.0 & 67.8 & 74.4 & 92 & 85.2 & 86.2 & 39.1 & 81.2 & 58.9 & 83.8 & 83.9 & 84.3 & 84.8 & 62.1 & 83.2 & 58.2 & 80.8 & 72.3 & 75.3 \\
ResNet38 [32] & 94.4 & 72.9 & 94.9 & 68.8 & 78.4 & 90.6 & 90.0 & 92.1 & 40.1 & 90.4 & 71.7 & 89.9 & 93.7 & 91.0 & 89.1 & 71.3 & 90.7 & 61.3 & 87.7 & 78.1 & 82.5 \\
PSPNet [37] & 91.8 & 71.9 & 94.7 & 71.2 & 75.8 & 95.2 & 89.9 & 95.9 & 39.3 & 90.7 & 71.7 & 90.5 & 94.5 & 88.8 & 89.6 & 72.8 & 89.6 & $\mathbf{6 4 . 0}$ & 85.1 & 76.3 & 82.6 \\
EncNet [34] & 94.1 & 69.2 & $\mathbf{9 6 . 3}$ & 76.7 & $\mathbf{8 6 . 2}$ & 96.3 & 90.7 & 94.2 & 38.8 & 90.7 & 73.3 & 90.0 & 92.5 & 88.8 & 87.9 & 68.7 & 92.6 & 59.0 & 86.4 & 73.4 & 82.9 \\
APCNet [11] & 95.8 & 75.8 & 84.5 & 76.0 & 80.6 & 96.9 & 90.0 & 96.0 & $\mathbf{4 2 . 0}$ & 93.7 & 75.4 & 91.6 & 95.0 & 90.5 & 89.3 & 75.8 & 92.8 & 61.9 & 88.9 & 79.6 & 84.2 \\
CFNet [35] & 95.7 & 71.9 & 95.0 & 76.3 & 82.8 & 94.8 & 90.0 & 95.9 & 37.1 & 92.6 & 73.0 & 93.4 & 94.6 & 89.6 & 88.4 & 74.9 & $\mathbf{9 5 . 2}$ & 63.2 & $\mathbf{8 9 . 7}$ & 78.2 & 84.2 \\
DMNet [10] & 96.1 & 77.3 & 94.1 & 72.8 & 78.1 & $\mathbf{9 7 . 1}$ & $\mathbf{9 2 . 7}$ & 96.4 & 39.8 & 91.4 & 75.5 & 92.7 & $\mathbf{9 5 . 8}$ & 91.0 & $\mathbf{9 0 . 3}$ & $\mathbf{7 6 . 6}$ & 94.1 & 62.1 & 85.5 & 77.6 & 84.4 \\
\hline Ours & $\mathbf{9 6 . 3}$ & 76.2 & 95.3 & $\mathbf{7 8 . 1}$ & 80.8 & 96.5 & 91.8 & $\mathbf{9 6 . 9}$ & 40.7 & $\mathbf{9 6 . 3}$ & $\mathbf{7 6 . 4}$ & $\mathbf{9 4 . 3}$ & $\mathbf{9 5 . 8}$ & $\mathbf{9 1 . 3}$ & 89.1 & 73.1 & 93.3 & 62.2 & 86.7 & $\mathbf{8 0 . 2}$ & $\mathbf{8 5 . 1}$ \\
\hline
\end{tabular}

validation sets. In Table 10, we illustrate the results of our CaC-Net and stateof-the-art methods on PASCAL VOC 2012 benchmark. We can observe that our CaC-Net yields mIOU $85.1 \%$ ont the test set, which outperforms other methods without $\mathrm{COCO}$ pre-training and achieves superior performance on most categories.

\subsection{Results on ADE20K}

ADE20K dataset [40] is a large-scale scene parsing dataset which provides 150 class categories, and consists of $20 \mathrm{~K}$ training images, $2 \mathrm{~K}$ validation and $3 \mathrm{~K}$ test samples. In this subsection, we carry out experiments on ADE20K to evaluate the effectiveness of our proposed CaC-Net. We train our CaC-Net with a ResNet101 backbone on the training set and evaluate the models on the validation set. Results of state-of-the-art methods on ADE20K are shown in Table 9. Our CaCNet with a ResNet101 backbone achieves $46.12 \%$ mIOU and outperforms all previous methods in mIoU with the same backbone. In Figure 4, we show some results of our predicted results and randomly select some representative channels of their feature maps in CaC-Net. In the visualized feature maps, the darker 

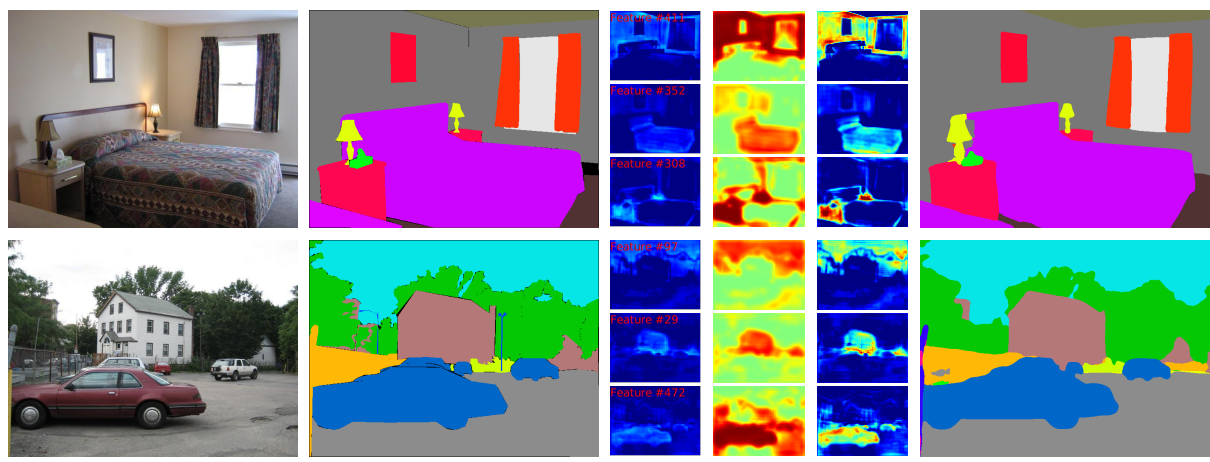

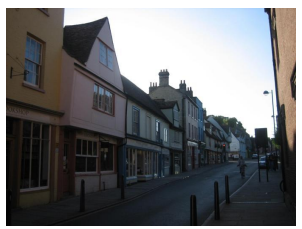

(a) Image

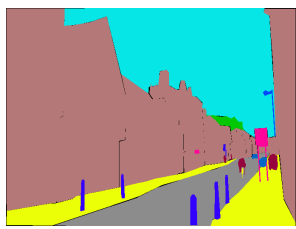

(b) Ground Truth

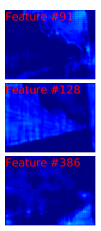

(c) $X$

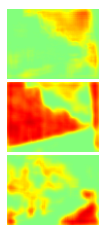

(d) $W$

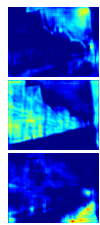

(e) $X^{\star}$

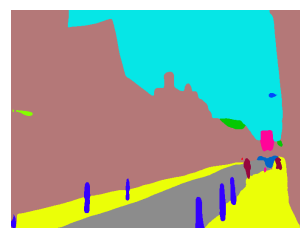

(f) $\mathrm{CaC}-\mathrm{Net}$

Fig. 4: Visualization results on ADE20K dataset. (a) The input images. (b) Ground truth label maps. (c)-(e) Some representative channels of the input feature maps $X$, the output feature maps $X^{\star}$ and the produced spatially-varying weighting factors $W$. In our CaC-Net, $X^{\star}=X \odot W$. (f) Results of our proposed CaC-Net

colors represent the larger values. We can observe that the spatially-varying weights produced by our $\mathrm{CaC}-\mathrm{Net}$ can efficiently highlight the class-dependent regions.

\section{Conclusion}

In this paper, we propose a novel network, CaC-Net, for semantic segmentation. The key innovation lies in the prediction of context-adaptive convolutional kernels to integrate both global context of the input semantic feature maps. Convolution with the predicted kernels leads to feature re-weighting maps that can effectively re-weight the feature maps in a spatially-varying manner. Extensive experiments demonstrate the outstanding performance of our proposed $\mathrm{CaC}$ Net, surpassing state-of-the-art segmentation methods on multiple datasets.

\section{References}

1. Arnab, A., Jayasumana, S., Zheng, S., Torr, P.H.: Higher order conditional random fields in deep neural networks. In: European Conference on Computer Vision. pp. 524-540. Springer (2016) 12

2. Badrinarayanan, V., Kendall, A., Cipolla, R.: Segnet: A deep convolutional encoder-decoder architecture for image segmentation. arXiv preprint arXiv:1511.00561 (2015) 12 
3. Bottou, L.: Large-scale machine learning with stochastic gradient descent. In: Proceedings of COMPSTAT'2010, pp. 177-186. Springer (2010) 8

4. Chen, L.C., Papandreou, G., Kokkinos, I., Murphy, K., Yuille, A.L.: Deeplab: Semantic image segmentation with deep convolutional nets, atrous convolution, and fully connected crfs. IEEE transactions on pattern analysis and machine intelligence 40(4), 834-848 (2017) 1, 3, 6, 8, 12, 13

5. Chen, L.C., Papandreou, G., Schroff, F., Adam, H.: Rethinking atrous convolution for semantic image segmentation. arXiv preprint arXiv:1706.05587 (2017) 1, 3

6. Everingham, M., Van Gool, L., Williams, C.K., Winn, J., Zisserman, A.: The pascal visual object classes (voc) challenge. International journal of computer vision 88(2), 303-338 (2010) 8, 12

7. Fu, J., Liu, J., Tian, H., Li, Y., Bao, Y., Fang, Z., Lu, H.: Dual attention network for scene segmentation. In: Proceedings of the IEEE Conference on Computer Vision and Pattern Recognition. pp. 3146-3154 (2019) 1, 3, 9, 12

8. Fu, J., Liu, J., Wang, Y., Li, Y., Bao, Y., Tang, J., Lu, H.: Adaptive context network for scene parsing. In: The IEEE International Conference on Computer Vision (ICCV) (October 2019) 12

9. Hariharan, B., Arbeláez, P., Girshick, R., Malik, J.: Hypercolumns for object segmentation and fine-grained localization. In: Proceedings of the IEEE conference on computer vision and pattern recognition. pp. 447-456 (2015) 12

10. He, J., Deng, Z., Qiao, Y.: Dynamic multi-scale filters for semantic segmentation. In: Proceedings of the IEEE International Conference on Computer Vision. pp. 3562-3572 (2019) 4, 8, 10, 12, 13

11. He, J., Deng, Z., Zhou, L., Wang, Y., Qiao, Y.: Adaptive pyramid context network for semantic segmentation. In: Proceedings of the IEEE Conference on Computer Vision and Pattern Recognition. pp. 7519-7528 (2019) 1, 3, 9, 10, 11, 12, 13

12. He, K., Zhang, X., Ren, S., Sun, J.: Deep residual learning for image recognition. In: Proceedings of the IEEE conference on computer vision and pattern recognition. pp. 770-778 (2016) 6, 8, 9

13. Hu, J., Shen, L., Sun, G.: Squeeze-and-excitation networks. In: Proceedings of the IEEE conference on computer vision and pattern recognition. pp. 7132-7141 (2018) $2,3,4,11$

14. Huang, Z., Wang, X., Huang, L., Huang, C., Wei, Y., Liu, W.: CCnet: Criss-cross attention for semantic segmentation. In: The IEEE International Conference on Computer Vision (ICCV) (October 2019) 12

15. Jia, X., De Brabandere, B., Tuytelaars, T., Gool, L.V.: Dynamic filter networks. In: Advances in Neural Information Processing Systems. pp. 667-675 (2016) 2, 4, 6

16. Li, X., Zhong, Z., Wu, J., Yang, Y., Lin, Z., Liu, H.: Expectation-maximization attention networks for semantic segmentation. In: The IEEE International Conference on Computer Vision (ICCV) (October 2019) 1

17. Lin, D., Ji, Y., Lischinski, D., Cohen-Or, D., Huang, H.: Multi-scale context intertwining for semantic segmentation. In: Proceedings of the European Conference on Computer Vision (ECCV). pp. 603-619 (2018) 12

18. Lin, G., Milan, A., Shen, C., Reid, I.: Refinenet: Multi-path refinement networks for high-resolution semantic segmentation. In: Proceedings of the IEEE conference on computer vision and pattern recognition. pp. 1925-1934 (2017) 12

19. Lin, G., Shen, C., Van Den Hengel, A., Reid, I.: Efficient piecewise training of deep structured models for semantic segmentation. In: Proceedings of the IEEE Conference on Computer Vision and Pattern Recognition. pp. 3194-3203 (2016) 12,13 
20. Liu, W., Rabinovich, A., Berg, A.C.: Parsenet: Looking wider to see better. arXiv preprint arXiv:1506.04579 (2015) 3

21. Liu, W., Rabinovich, A., Berg, A.C.: Parsenet: Looking wider to see better. arXiv preprint arXiv:1506.04579 (2015) 12

22. Liu, Z., Li, X., Luo, P., Loy, C.C., Tang, X.: Semantic image segmentation via deep parsing network. In: Proceedings of the IEEE International Conference on Computer Vision. pp. 1377-1385 (2015) 13

23. Long, J., Shelhamer, E., Darrell, T.: Fully convolutional networks for semantic segmentation. In: Proceedings of the IEEE conference on computer vision and pattern recognition. pp. 3431-3440 (2015) 1, 3, 9, 12, 13

24. Mildenhall, B., Barron, J.T., Chen, J., Sharlet, D., Ng, R., Carroll, R.: Burst denoising with kernel prediction networks. In: Proceedings of the IEEE Conference on Computer Vision and Pattern Recognition. pp. 2502-2510 (2018) 4

25. Mottaghi, R., Chen, X., Liu, X., Cho, N.G., Lee, S.W., Fidler, S., Urtasun, R., Yuille, A.: The role of context for object detection and semantic segmentation in the wild. In: Proceedings of the IEEE Conference on Computer Vision and Pattern Recognition. pp. 891-898 (2014) 8, 9

26. Niklaus, S., Mai, L., Liu, F.: Video frame interpolation via adaptive separable convolution. In: Proceedings of the IEEE International Conference on Computer Vision. pp. 261-270 (2017) 4

27. Noh, H., Hong, S., Han, B.: Learning deconvolution network for semantic segmentation. In: Proceedings of the IEEE international conference on computer vision. pp. 1520-1528 (2015) 13

28. Russakovsky, O., Deng, J., Su, H., Krause, J., Satheesh, S., Ma, S., Huang, Z., Karpathy, A., Khosla, A., Bernstein, M., et al.: Imagenet large scale visual recognition challenge. International journal of computer vision 115(3), 211-252 (2015) 8

29. Su, H., Jampani, V., Sun, D., Gallo, O., Learned-Miller, E., Kautz, J.: Pixeladaptive convolutional neural networks. In: Proceedings of the IEEE Conference on Computer Vision and Pattern Recognition. pp. 11166-11175 (2019) 4

30. Takikawa, T., Acuna, D., Jampani, V., Fidler, S.: Gated-scnn: Gated shape cnns for semantic segmentation. In: Proceedings of the IEEE International Conference on Computer Vision. pp. 5229-5238 (2019) 1

31. Vaswani, A., Shazeer, N., Parmar, N., Uszkoreit, J., Jones, L., Gomez, A.N., Kaiser, Ł., Polosukhin, I.: Attention is all you need. In: Advances in neural information processing systems. pp. 5998-6008 (2017) 8

32. Wu, Z., Shen, C., Hengel, A.v.d.: Wider or deeper: Revisiting the resnet model for visual recognition. arXiv preprint arXiv:1611.10080 (2016) 13

33. Yu, F., Koltun, V., Funkhouser, T.: Dilated residual networks. In: Proceedings of the IEEE conference on computer vision and pattern recognition. pp. 472-480 (2017) 6

34. Zhang, H., Dana, K., Shi, J., Zhang, Z., Wang, X., Tyagi, A., Agrawal, A.: Context encoding for semantic segmentation. In: The IEEE Conference on Computer Vision and Pattern Recognition (CVPR) (June 2018) 1, 2, 3, 4, 6, 8, 9, 11, 12, 13

35. Zhang, H., Zhang, H., Wang, C., Xie, J.: Co-occurrent features in semantic segmentation. In: The IEEE Conference on Computer Vision and Pattern Recognition (CVPR) (2019) 1, 3, 8, 9, 11, 12, 13

36. Zhang, R., Tang, S., Zhang, Y., Li, J., Yan, S.: Scale-adaptive convolutions for scene parsing. In: Proceedings of the IEEE International Conference on Computer Vision. pp. 2031-2039 (2017) 4, 12 
37. Zhao, H., Shi, J., Qi, X., Wang, X., Jia, J.: Pyramid scene parsing network. In: Proceedings of the IEEE conference on computer vision and pattern recognition. pp. 2881-2890 (2017) 1, 3, 8, 9, 12, 13

38. Zhao, H., Zhang, Y., Liu, S., Shi, J., Change Loy, C., Lin, D., Jia, J.: PSANet: Point-wise spatial attention network for scene parsing. In: Proceedings of the European Conference on Computer Vision (ECCV). pp. 267-283 (2018) 3, 12

39. Zheng, S., Jayasumana, S., Romera-Paredes, B., Vineet, V., Su, Z., Du, D., Huang, C., Torr, P.H.: Conditional random fields as recurrent neural networks. In: Proceedings of the IEEE international conference on computer vision. pp. 1529-1537 (2015) 12, 13

40. Zhou, B., Zhao, H., Puig, X., Fidler, S., Barriuso, A., Torralba, A.: Scene parsing through ade20k dataset. In: Proceedings of the IEEE conference on computer vision and pattern recognition. pp. 633-641 (2017) 8, 12, 13

41. Zhu, Z., Xu, M., Bai, S., Huang, T., Bai, X.: Asymmetric non-local neural networks for semantic segmentation. In: Proceedings of the IEEE International Conference on Computer Vision. pp. 593-602 (2019) 12 